\title{
Multiple sclerosis associated with syringomyelia
}

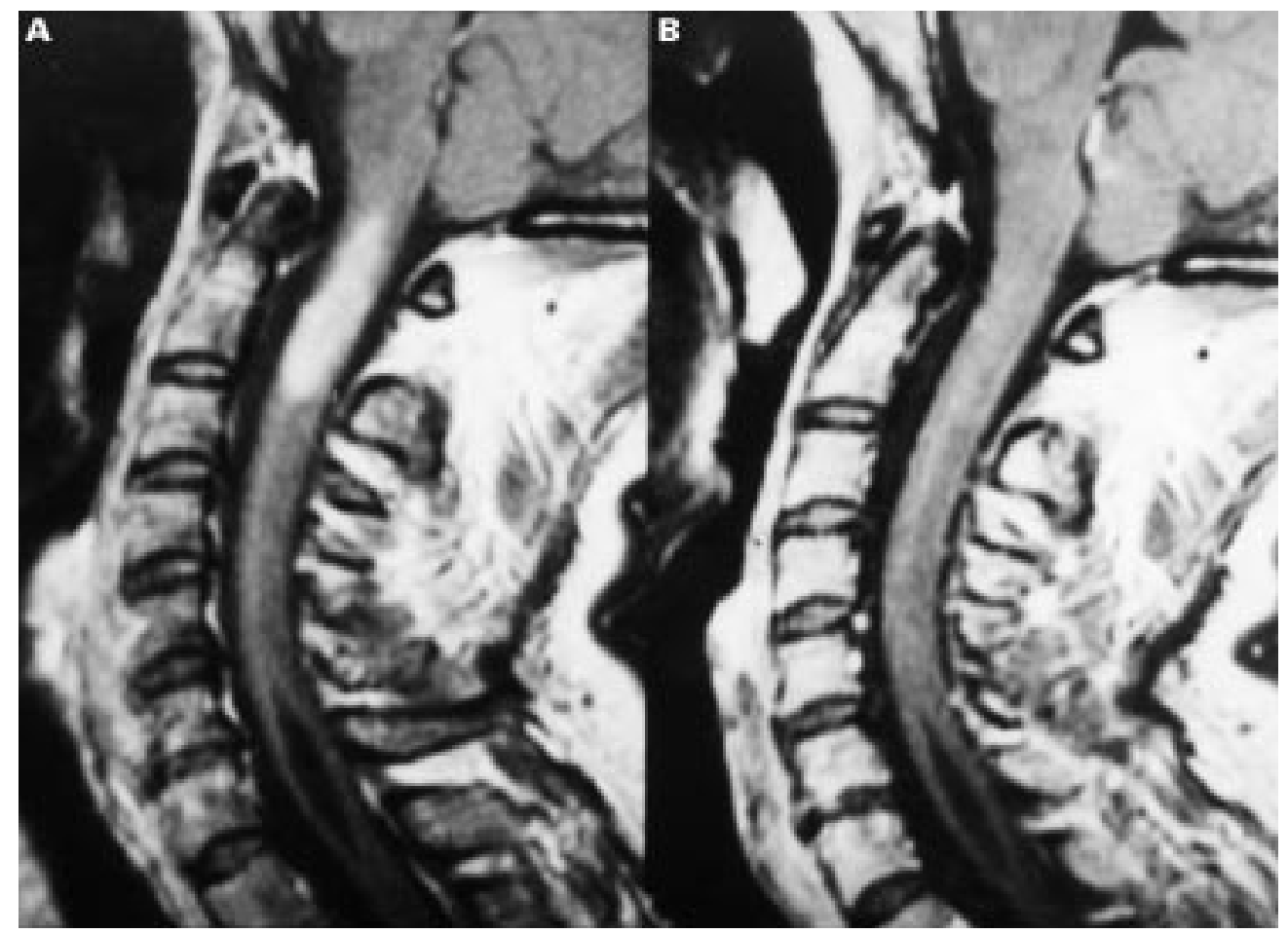

A 41 year old woman experienced subacute onset of weakness in her right arm, hypoesthesia of the left limbs, urinary urgency, and radicular-like pain in her third right cervical root territory. The neurological examination disclosed a right Brown-Séquard syndrome at the C3 level and horizontal disconjugated nystagmus gazing to the right. Spinal magnetic resonance (MR) (figure A) showed a large, proton density and T2 weighted hyperintense and Gd enhancing intramedullary lesion extending from the level of $\mathrm{C} 1$ to $\mathrm{C} 3$ discs encompassing the central canal, associated with a distal, not Gd enhancing small syrinx centrally located and extending from C5 to C6 disks. There was no evidence of spinal cord tumour or of Chiari malformation. Brain MR showed bilateral multiple, proton density and T2 weighted high intensity signal abnormalities in both brain and cerebellar hemispheres consistent with multiple sclerosis lesions, with no Gd enhancement. Visual evoked potentials showed increased latency of the P100 component after visual stimulation of the right eye. Cerebrospinal fluid isoelectrofocusing disclosed four oligoclonal bands. A diagnosis of laboratory supported definite multiple sclerosis was made according to Poser's criteria and $1 \mathrm{~g}$ intravenous methylprednisolone daily for 5 days was administered. Two months later the symptoms subsided. MRI of the cervical cord showed reduction of the demyelinating lesion, with loss of $\mathrm{Gd}$ enhancement. The syringomy- elic cavity was substantially unchanged. Six months after onset the symptoms remitted. Proton density, T2 weighted, and Gd enhanced MRI showed disappearance of the demyelinating lesion and a slight reduction in size of the cavity (figure B).

Very few radiographically documented hydrosyringomyelia in patients with multiple sclerosis have been reported in the literature since the first description in $1985^{1}$. Our findings suggest that spinal syringomyelic cavities may develop and fluctuate during the course of multiple sclerosis as a consequence of CSF dynamics imbalance due to multiple sclerosis lesions affecting critical areas of the spinal cord. ${ }^{2}$

STEFANO SOTGIU GIANPIETRO SECHI MAURA PUGLIATTI PAOLA VALENTI GIULIO ROSATI

Institute of Clinical Neurology, University of Sassari, Italy

ANTONIO ACHENE

MAURIZIO CONTI

Institute of Radiology, University of Sassari, Italy

Correspondence to: Dr Stefano Sotgiu, Clinica Neurologica, Università di Sassari, Viale San Pietro 10, 07100 Sassari, Italy. Telephone 0039079228 231; fax 0039079228 423; email. clineuro@ssnet.it

1 Kwee IL, Nakada T. Syrinx formation in multiple sclerosis. Brf Radiol 1985;58:1206-8.

2 Tartaglino LM, Friedman DP, Flanders AE, et al. Multiple sclerosis in the spinal cord: MR appearance and correlation with clinical parameters. Radiology 1995;195:725-32. 